\title{
What to call arrhythmias?
}

\author{
DENNIS KRIKLER \\ From the Division of Cardiovascular Disease, Royal Postgraduate Medical School, Hammersmith Hospital, \\ London
}

The problem of definition of disorders of cardiac rhythm and conduction has received fresh consideration largely because of advances in electrophysiological techniques. Recently, two bodies have tried to clarify terminology, and their proceedings have been published in close succession. The American College of Cardiology (Tenth Bethesda Conference, 1978) held a meeting on 'Optimal Electrocardiography' in 1977, during which many other aspects of electrocardiography were also discussed. Task Force I, one of the committees that met during this conference, was devoted to standardisation of terminology and interpretation. Parallel with this American venture, a committee set up by the World Health Organisation and the International Society of Cardiology has also considered definitions of terms related to cardiac rhythm (WHO/ISC Task Force, 1978). Both groups explain why some terms are to be preferred and others discouraged; as the introduction to the international report stresses, the motivation is 'the need for common language in electrocardiology.'

At once we are confronted with a recurrent problem; what overall term should be used to describe disorders of rhythm and conduction? In keeping with the reasoned exposition by Papp (1969), both of the present reports unhesitatingly select 'arrhythmia'. 'Dysrhythmia' is a more recent neologism, for which there is no good justification. It is used, by its exponents, to mean 'absence of physiological rhythm', the sense in which 'arrhythmia' has been used for more than 100 years (Scherf and Schott, 1953). The WHO/ISC Task Force defines arrhythmia as 'any cardiac rhythm other than normal sinus rhythm. Such a rhythm may be either of sinus or ectopic origin, and either regular or irregular. An arrhythmia may be due to a disturbance in impulse formation or conduction, or both.' Thus those who attempt to introduce 'dysrhythmia' merely compound confusion, especially when, as one tends to see more often-though not ex- clusively-in general than in cardiac journals, the words 'arrhythmia' and 'dysrhythmia' appear synonymously in the same article. 'Arrhythmia' conforms with classic Greek usage; as Papp said nearly 10 years ago, 'the pioneers of cardiac arrhythmias had as good or better classical knowledge as many of us.' Here and elsewhere we should heed his advice not to sacrifice international understanding to 'false linguistic purism'.

Fortunately the terms preferred by the two recent 'task forces' are compatible, but they deserve careful consideration. What is the justification for rules? Are we being too rigid in asking ourselves to stick to them? Those who use indexes, not to mention the hardworking compilers, must not be frustrated because of unnecessary synonyms and the corollary that even with careful scrutiny an important subject may be missed because it is listed according to the individual whim of an author. To this extent we must accept discipline, so that we can communicate successfully with one another. Anatomically it should be easiest to be precise but there has been much confusion and semantic difficulty. With this has gone uncertainty about the criteria for interpretation of electrocardiographic appearances said to reflect a variety of disorders of rhythm and conduction. Recent collaborative work has clarified the anatomy of the conducting tissues and provided useful definitions-and, incidentally, confirmed many early descriptions (Anderson and Becker, 1978).

Coming back to electrocardiography, as computeraided interpretation becomes more widely used, the development of a proper data base requires consistency of language. As a result of the Tenth Bethesda Conference, an ad hoc committee of the American College of Cardiology and the American Heart Association is planned, so as to ensure coordination. The WHO/ISC Task Force will shortly publish a classification of cardiac arrhythmias and conduction disturbances based on the 
and not altered thereafter during the examination. Swept gain was not used. All investigations were recorded on three-quarter inch tape using a Sony U-matic video tape recorder (VO 2631). An internally derived grey scale was superimposed on each display.

\section{PROCESSING OF IMAGES}

Three types of display were investigated: (1) Standard black and white; (2) simple colour coding -here, the amplitude range of the A mode display was split into seven levels, plus zero (black). Each amplitude level was represented as a single colour in the sequence: cyan, green, yellow, red, magenta, blue, and white, the last corresponding to maximum amplitude. (3) Image processing, when both colour and brightness were modulated, using a Brompton Encoder (manufactured by Alltek Hospital Supplies, Ltd, Shepperton, Middlesex, UK). In this approach, the same colour sequence was used, but the luminosity of each colour was varied within each level. This allowed a continuous range of echo amplitude to be displayed, and also meant that regions of low echo amplitude appeared with low brightness on the screen, and so were less conspicuous on the final image. This resulted in preservation of the original amplitude perspective. These three methods of displaying grey scale are compared in Fig. 1.

\section{ANALYSIS OF IMAGES}

Since absolute figures for echo amplitude were not available, relative figures were used. Zero amplitude was taken as black. The maximum value (white) was established in two ways: (1) By taking the maximum level of the internal standard grey scale generated by the echocardiograph, corresponding to a TV
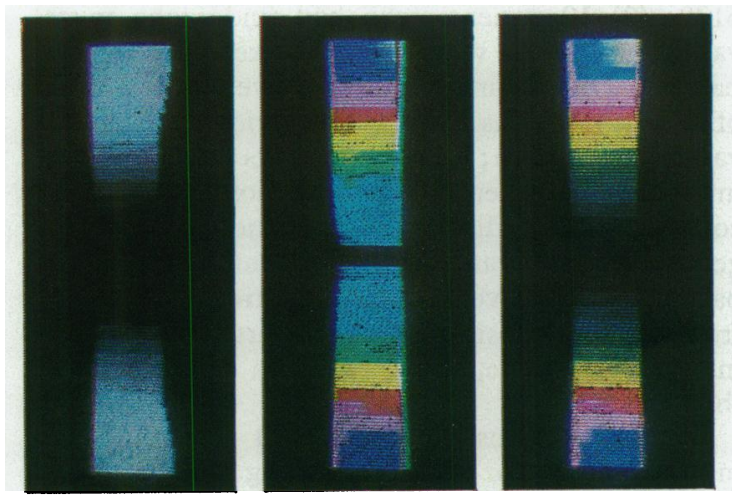

Fig. 1 Comparison of three methods of display of standard grey scale. (a) Standard black and white, $(b)$ simple colour coding, and (c) processed image, with modulation of both hue and luminosity. video output of 0.7 volt; and (2) by taking the interface immediately behind the left ventricular posterior wall on the parasternal long axis view as 100 per cent. This interface corresponds to the junction between pericardium and air-containing lung, and was consistently found in all patients examined. In order to gain some idea of the consistency of these two methods, the calibration of the processor, expressed in arbitrary units, necessary to establish the amplitude range by each was noted.

Measurements of echo intensity were made from stop frame end-diastolic images, and the dominant colour of structures was noted, and expressed as a percentage, white being 100 per cent. Measurements were made of septal and posterior wall, myocardium, central fibrous body, and aortic and mitral valves. After mitral valve replacement, the intensity of echoes from the ring and poppet of the StarrEdwards prosthesis or the plastic stent of the Hancock xenograft was noted. Values obtained from the image in the parasternal long axis view were then compared with those of the same structure in the apical four chamber view, when it was adequately visualised in both. After the dominant level of echo intensity from any structure had been recorded, a qualitative note was made of abnormalities of texture or distribution of amplitude when appropriate.

\section{STATISTICAL METHODS}

Mean values between groups of patients were compared by Student's $t$ test. Linear regression analysis was by the method of least squares.

\section{Results}

NORMAL SUBJECTS

Apart from the posterior heart border (taken as $100 \%$ ), the most densely reflecting structure in the normal heart proved to be the central fibrous body, with a value of $64 \pm 5$ per cent. Mitral and aortic valves reflected at approximately half this level, at $35 \pm 5$ and $36 \pm 8$ per cent, respectively. The intensity of the interventricular septum, $33 \pm 8$ per cent, was significantly greater than that of the posterior wall, $23 \pm 6$ per cent $(p<0.05)$. Values from endocardium were not significantly different from those of underlying myocardium. An exception to this was seen in the minor axis view of the septum, when the endo- and epicardium in a small region perpendicular to the ultrasound beam reflected at approximately two levels higher than surrounding tissue. In addition to this, there were occasional brightly reflecting targets on the normal endocardium, which were particularly obvious at end- 


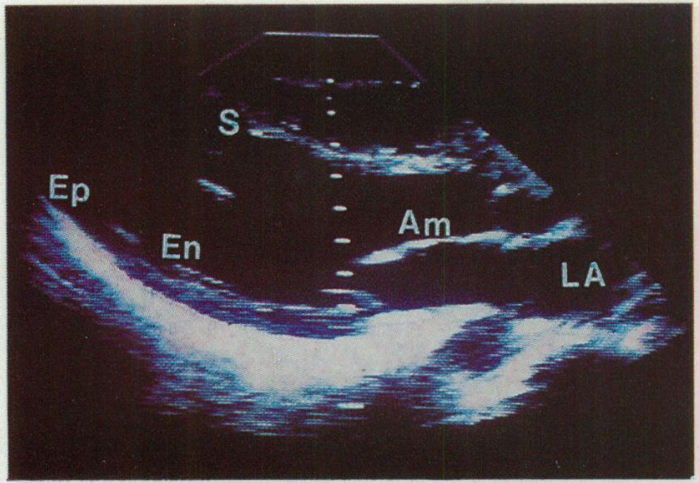

Fig. 2a

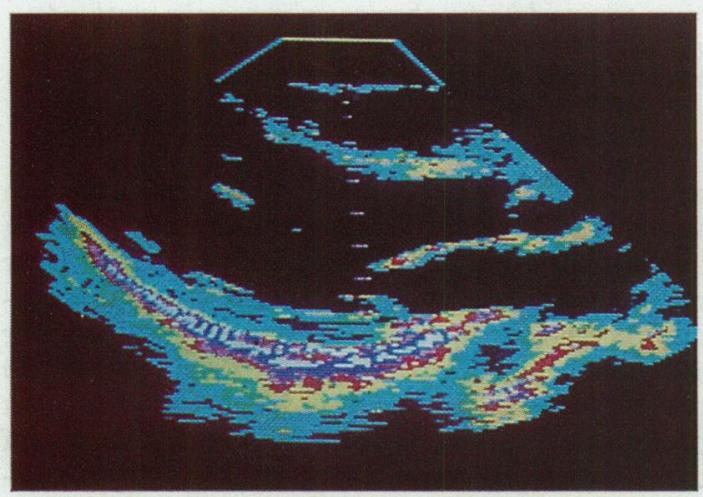

Fig. 2b

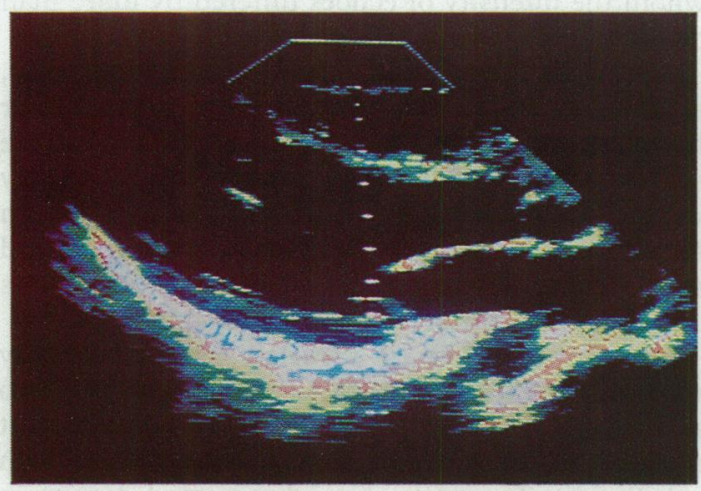

Fig. 2c

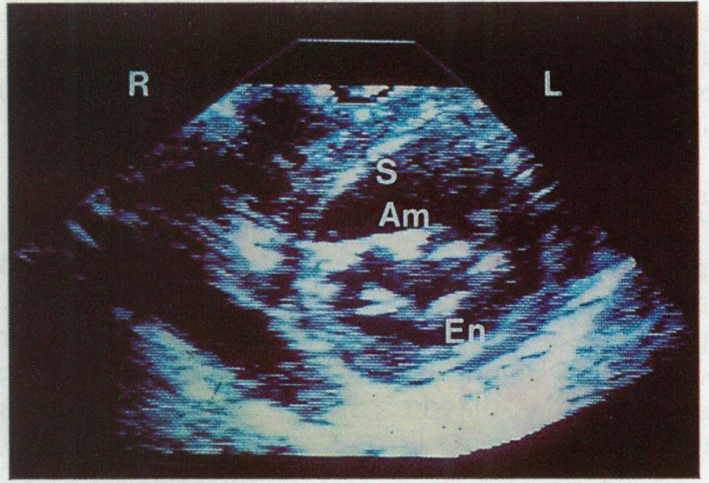

Fig. 3a

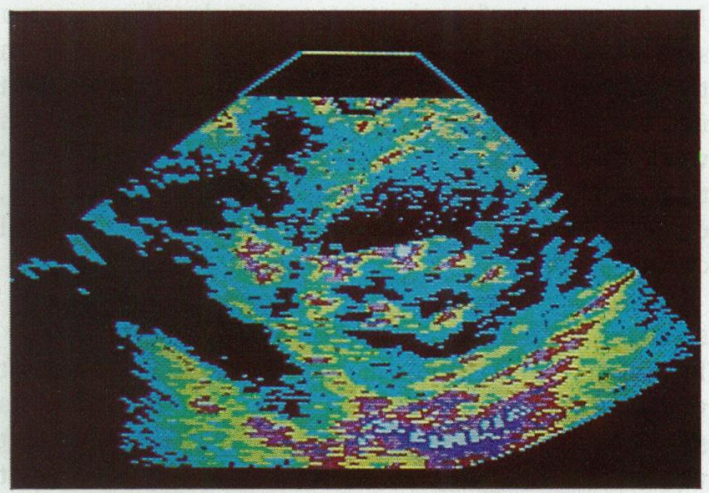

Fig. 3b

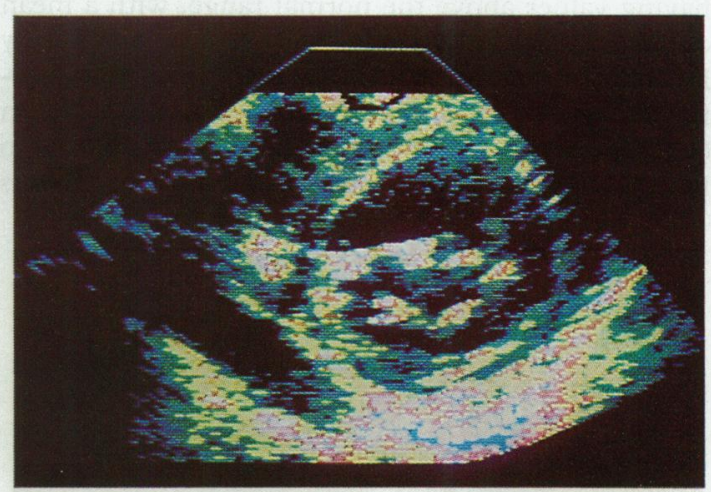

Fig. 3c

Fig. 2 Normal heart, parasternal long axis view, showing interventricular septum (S), anterior cusp of mitral valve $(A m)$, left atrium $(L A)$, and endo $(E n)$ and epicardium $(E p)$ of posterior left ventricular wall. Standard grey scale image is shown in (a), simple colour coded image in $(b)$, and processed image in $(c)$. Note that normal left ventricular posterior wall and septal myocardium both reflect at relatively low intensity (cyan or green).

Fig. 3 Rheumatic mitral valve disease, parasternal short axis view. Abbreviations and image processing as Fig. 2. Note (1), highly reflecting areas in anterior cusp (100\%), and (2) laminar regions of increased reflection in septal and posterior wall myocardium. Comparison of $(b)$ and $(c)$ shows effect of simple colour coding versus processing. Right $(R)$ and left $(L)$ sides of image are indicated. 
systole. In Fig. 2 are shown representative examples from a normal subject of the black and white (2a), simple colour coded (2b), and processed (2c) images.

\section{PROSTHETIC MITRAL VALVES}

The metal sewing ring of the Starr-Edwards prosthesis consistently reflected at 100 per cent, and the silastic poppet at $44 \pm 5$ per cent. Values for the plastic stent of the Hancock xenograft, $75 \pm 4$ per cent, were significantly lower than those for the sewing ring of the Starr-Edwards prosthesis $(\mathrm{p}<0.01)$.

\section{RHEUMATIC MITRAL VALVE DISEASE}

In the group of patients as a whole, there was a significant increase in the intensity of the echo from the anterior cusp ( $54 \pm 11 \%)$, which was greater than normal ( $p<0.01)$ (Fig. 3). When individual patients were considered, however, only 10 had levels above the upper 95 per cent confidence limit of normal, eight of whom had radiological evidence of mitral calcification.

\section{LEFT VENTRICULAR DISEASE}

Increased amplitude of reflection from the left ventricular wall was common in the patients studied. In those with severe impairment of left ventricular function from coronary artery disease, it was particularly common in the septum, all patients having values above the normal range, with a mean of $71 \pm 11$ per cent (Fig. 4). It was frequently layered in both septum and free wall, with preferential occurrence in the subendocardial region (Fig. 5). The basal region of the septum seemed to be more affected than the apical in the patients

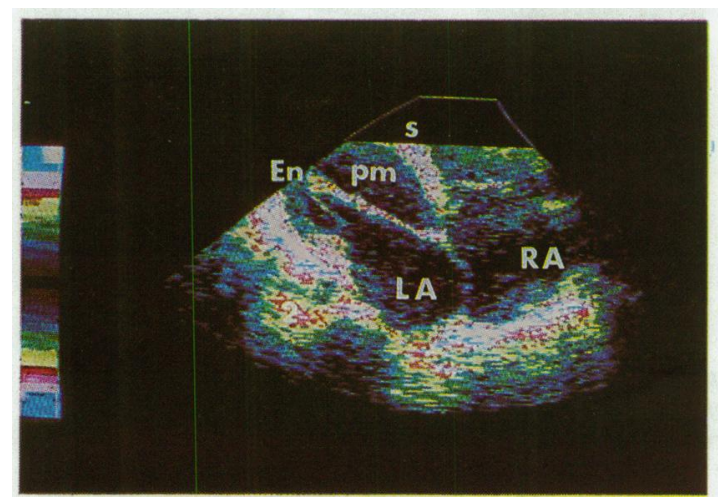

Fig. 4 Septal fibrosis, apical four chamber view, processed image. Abbreviations as Fig. 3. RA, right atrium; LA, left atrium, pm, papillary muscle. Note that there is a striking increase in septal intensity to 100 per cent, while posterior wall endocarium is normal.

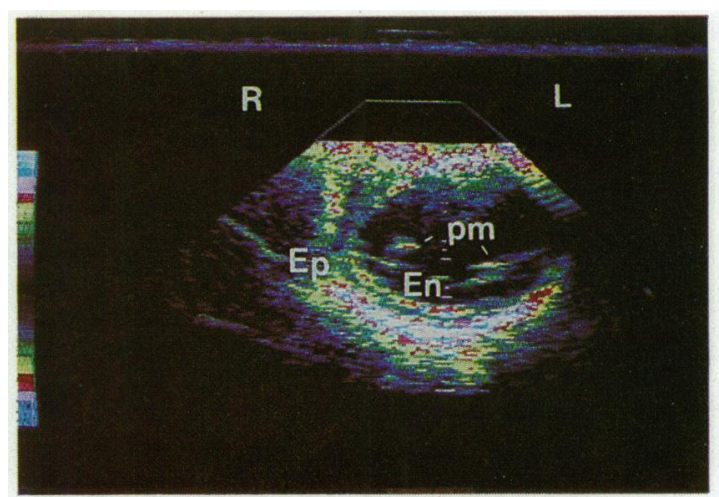

Fig. 5 Ischaemic heart disease, parasternal minor axis view, processed image, showing an increase in amplitude of reflection from the subendocardial region of the posterior wall. Abbreviations as Fig. 4.

studied. Involvement of the septum was also apparent in the apical four chamber view, showing that the apparent increase in the parasternal view was not simply a function of nearness to the transducer. By contrast, involvement of the posterior wall was less obvious, with only eight of the 15 patients showing values above the 95 per cent confidence limits of normal. Involvement of one of the two papillary muscles, apparent in the parasternal minor axis view, was seen in three patients, in whom the intensity of echoes differed by more than two levels (Fig. 6).

In patients with left ventricular hypertrophy, areas of increased echo intensity were seen rather less frequently, five patients having values for the septum, and eight for the posterior wall which lay within the normal range. Increased density was seen focally in two patients with aortic stenosis, and subendocardially in three, two with aortic stenosis and one with hypertrophic cardiomyopathy.

Abnormalities of left ventricular echo intensity were also apparent in the other patient groups. Two of the patients with mitral valve replacement showed an increase in septal density. Six with calcific mitral stenosis showed increased density in the posterior wall, and 12 an increase in that of the septum (Fig. 3c). These changes were not seen in patients without mitral calcification.

COMPARISON OF CALIBRATION METHODS

The mean value of calibration setting of the processor required when the maximum level of the internal grey scale was used to determine 100 per cent amplitude was $567 \pm 44$ units. When calibration was based on the intensity of the posterior heart border, it was $604 \pm 61$ units. This difference, 


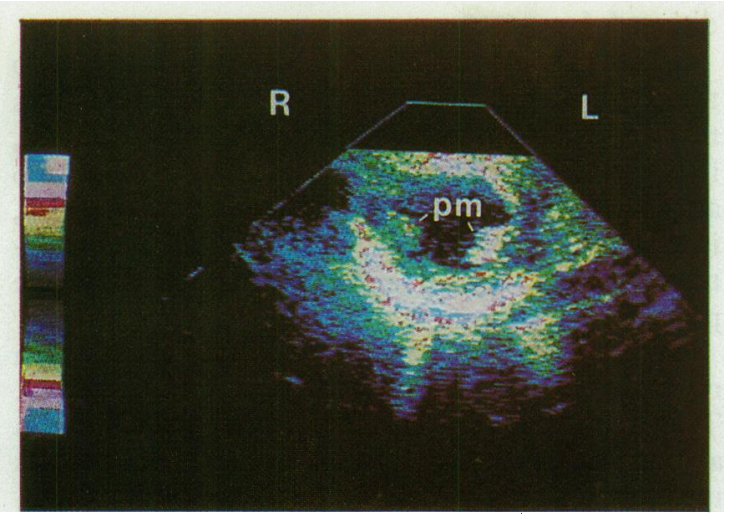

Fig. 6 Ischaemic heart disease, parasternal minor axis view, processed image, showing an increase in amplitude of reflection from the left sided papillary muscle (up to $100 \%)$, significantly greater than that from the right (less than 50\%). Abbreviations as Fig. 4.

corresponding to approximately half an intensity level, was consistent between the two methods $(\mathrm{p}<0.01)$.

\section{EFFECT OF BEAM DIRECTION ON}

\section{AMPLITUDE MEASUREMENTS}

The intensity of the echoes from 40 structures (posterior wall, septum, mitral valve) in 16 patients was compared in the parasternal long axis and apical four chamber views. Values of echo intensity in the parasternal view ranged from 15 to 100 per cent.

There was close correlation between measurements made from the two sites, given by the equation:

Amplitude (PAS) $=0.96$

Amplitude (4C) $+0.86 \%$, where Amplitude (PAS) and Amplitude (4C) represent the respective amplitudes in the parasternal and apical four chamber views. The correlation coefficient was $0.95, \mathrm{~F}$ value was 312 , and the standard error of the estimate 8 per cent. The slope of the regression line did not differ significantly from one nor the intercept from zero.

\section{Discussion}

Factors affecting the amplitude of echoes returning from biological tissues are complex. This field has been reviewed in detail by Wells. ${ }^{1}$ Two main mechanisms are involved. The first is progressive attenuation of ultrasound as it passes through blood or tissue. This increases with path length, interfaces encountered, and the frequency of the ultrasound used. This attenuation was originally thought to be the result of dissipation of ultrasound energy by viscous forces, but this view does not account for its observed extent, and is no longer tenable. A more satisfactory explanation has been to ascribe the energy loss to a group of processes known collectively as "relaxation", by which the incident energy is redistributed within the molecules of the tissue through which it passes. This process takes a finite time, and the resulting delay leads to the generation of out-of-phase energy which interferes with the incident wave so that absorption occurs. It appears that in biological tissues, multiple time intervals and energy levels are involved, so that the process becomes one of great complexity. Loss of energy may also occur by dissipation as heat. The second factor determining the amplitude of the returning echo is the nature of the reflection at the target itself, which depends on its size, shape, and internal structure. If the target is large compared with the wavelength of ultrasound used, then simple laws of reflection apply, and the proportion of returning energy is determined by the accoustic impedance change between the two structures forming the interface, and its direction by the angle of incidence. Using a pulse echo technique, therefore, such specular interfaces are detected when they are perpendicular to the ultrasound beam. Much more commonly, however, the target is small compared with the wavelength of the ultrasound. In these circumstances, the ultrasound is scattered over a much wider angle rather than if it had undergone simple reflection, in a manner dependent on wavelength and internal structure. In view of the complexity of the determinants of echo amplitude, therefore, it seems reasonable to proceed empirically, rather than to insist on a precise theoretical basis for each stage of clinical investigation.

If regional differences in amplitude are to be appreciated, it is desirable that they are displayed with the original image. Even if grey scale is well preserved by the equipment and recording system used, the number of levels that can be differentiated by the eye is small. If two regions of similar, but not identical level are contiguous, the difference can usually be discerned, but if they are separated by black or white areas, this is no longer the case. On the other hand, grey scale has the advantage that areas of greatest amplitude, in appearing brightest on the display, are most prominent to the eye. The obvious alternative to grey scale is to use colour coding of amplitude, but this proved unsatisfactory because of the low information level and because attention was distracted from movement or boundaries by bright colours from regions of low amplitude, making them obtrusive (Fig. 3b). A combination of luminosity and colour processing, however, made it possible to appreciate the addi- 
tional information conveyed by colour certainly without loss, and possibly with enhancement of image quality (Fig. $3 \mathrm{c}$ ). As has previously proved to be the case with grey scale, the moving display appeared more informative and attractive in comparison with stop frames.

The present study suggests that clinically useful measurements of regional echo amplitude can be made in man. The range of values for normal myocardium and valve was narrow. In patients with prosthetic valves, the amplitude of the echo produced by a foreign substance of known and constant properties can be studied in vivo. Again, values from different patients fell within a narrow range, indicating that the methods used for setting up the range of the amplitude scale allowed useful comparison between subjects. Patients with rheumatic mitral valve disease are known to have fibrosis or calcification of the anterior cusp, so that they may be taken as an example of unequivocally abnormal tissue. Again, the method gave consistent results, showing an increase in reflection, particularly when calcification was present.

There is little information about regional abnormalities of echo amplitude occurring in left ventricular disease. A subjective increase in the intensity of septal echoes caused by scarring has been described by Rasmussen et al., ${ }^{2}$ and in association with papillary muscle dysfunction by Grison et $a .^{3}$ By contrast, discrete sonolucent areas were noted by Werner et al. ${ }^{4}$ after myocardial infarction. Tanaka and Terasawa, ${ }^{5}$ using a method capable of making objective measurements, described fibrotic changes caused by endocarditis, which were associated with a 5-10 dB increase in echo amplitude. More severe scarring led to a greater increase, so that echo intensity was at the same level as that arising from the pericardium. These findings were confirmed by biopsy or necropsy. The present preliminary observations confirm that objective measurements can be made of regions of increased echo intensity in the left ventricle whose function has been significantly impaired by coronary artery disease. They were particularly common in the septum and anterior wall, but were seen elsewhere, including the papillary muscles. They were either full or partial thickness, and frequently lay alongside rather than coinciding with areas showing maximum disturbance of wall movement. In about half the patients with left ventricular hypertrophy, regional echo amplitude was normal. In the remainder, either a more generalised granular appearance or localisation to the subendocardial region was seen. Though we have no direct evidence in the present patients, these findings coincide with previous histological descriptions of fibrosis in these con- ditions. ${ }^{6-8}$

There are a number of self-evident limitations to the clinical application of these results. The first is that absorption of ultrasound, and thus path length, is likely to have been a major factor affecting echo amplitude. This was apparent in normal subjects, in whom values of intensity obtained from the septum were consistently greater than those from the posterior wall.

Comparisons in patients should thus be made with normal values from the same region of the heart. This limitation is likely to be accentuated if there is a proximal interface which absorbs or reflects ultrasound strongly such as a thickened pericardium. It is also clear that our results apply to adults only, and need not necessarily refer to infants or children in whom path length is short and near field corruption a potential problem. Since tissue penetration is also a function of ultrasound frequency, echo amplitude from deeper structures will be more significantly reduced if ultrasound of 5 rather than $3 \mathrm{MHz}$ is used. Another potential limitation is that the direction from which a target is viewed might affect echo amplitude. This would be particularly important if reflection were specular. In practice, this did not appear to be the case, since values of intensity derived from parasternal and apical views were virtually identical, though small differences might have been present amounting to less than one of the amplitude levels used in the present study. It is possible, however, that the slight increase in septal amplitude noted in the septum immediately below the transducer in the minor axis view might have been a manifestation of specular reflection. Nevertheless, the small effect of beam direction has practical significance in clinical echocardiography, where the assumption that the ultrasound beam must have struck an interface with an angle of incidence approximating to perpendicular if satisfactory echoes are recorded has been used as a basis for geometrical models of intracardiac structures. The relation with angle of incidence may well merit further study, since detection of dependence of echo amplitude on beam direction might be a means of obtaining information about tissue structure, as has proved the case with liver and spleen. ${ }^{9}$ Estimates of echo amplitude in different parts of the same ventricle, and comparisons between patients both depend on the method used to calibrate the colour scale. We were encouraged, therefore, by the close agreement between the two approaches used, one based on an internal and the other on an external standard.

These results suggest that with suitable image processing, information about the amplitude of echoes arising from intracardiac structures can be 
superimposed on a grey scale display as colour without degrading image quality. The method proved satisfactory in demonstrating increased reflection from prostheses and rheumatic mitral valves, while observations in patients with left ventricular disease were compatible with previous histological observations. Though the theoretical basis of the method cannot yet be stated precisely, its implementation appears to extend currently available echocardiographic methods into a region of potential clinical significance.

\section{References}

1 Wells PNT. Biomedical ultrasonics. London: Academic Press, 1977: 110-37.

2 Rasmussen S, Corya BC, Feigenbaum H, Knoebel SB. Detection of myocardial scar tissue by $\mathrm{M}$-mode echocardiography. Circulation 1978; 57: 230-7.

3 Grison D, Dumeny P, Kagan L, Sacrez A. L'echographie uni et bidimensionelle dans les dysfonctionnements des piliers d'origine ischemique (abstract VIII) European Congress of Cardiology. European Society of Cardiology, 1980; 109.

4 Werner JA, Pearlman AS, Janko C. Pathophysiologic implications of a new echocardiographic finding in acute myocardial infarction. Abstract VIII Europ Congr Cardiol 1980; 76.

5 Tanaka M, Terasawa H. Echocardiography: evaluation of tissue character in myocardium. Fpn Circ $\mathcal{F}$ 1979; 43: $367-76$.

6 Harrison CV, Wood P. Hypertensive and ischaemic heart disease: a comparative clinical and pathological study. Br Heart f 1949; 11: 205-29.

7 Davies MJ, Fulton WFM, Robertson WB. The relation of coronary thrombosis to ischaemic myocardial necrosis. F Pathol 1979; 127: 99-110.

8 Snow PJD, Jones AM, Daber KS. Coronary disease: a pathological study. Br Heart $\mathcal{F} 1955$; 17: 503-10.

9 Nicholas D. Pattern recognition and diffraction analysis. In: Hill $\mathrm{CR}$, Alvisi $\mathrm{C}$, eds. Investigative ultrasonology. 1. Technical advances. Tunbridge Wells: Pitman Medical, 1980: 97-9.

Requests for reprints to Dr D G Gibson, Brompton Hospital, Fulham Road, London SW3 6HP. 